\title{
ANÁLISIS DEL CASO DEL RIOCIGUAT EN LA JUSTICIA CONSTITUCIONAL COSTARRICENSE'
}

\section{ANALYSIS OF THE RIOCIGUAT CASE IN COSTA RICA'S CONSTITUTIONAL JUSTICE SYSTEM}

\section{Cristina Carpio Alvarado² - Freddy Arias Mora ${ }^{3}$}

DOI: https://doi.org/10.37767/2591-3476(2021)02

Fecha de envío: 03.03.2021

Fecha de aceptación: 18.06.2021

\section{RESUMEN:}

La judicialización para que el sistema de salud público provea de medicamentos a pacientes en Costa Rica es cada vez más frecuente. La solución de estos casos en su mayoría le corresponde al tribunal constitucional. El presente artículo es un estudio cualitativo, analítico y descriptivo basado en el acceso al medicamento Riociguat para el tratamiento de pacientes con hipertensión pulmonar tromboembólica. Se analiza la totalidad de resoluciones sobre el Riociguat para el caso costarricense y una referencia a profundidad de la sentencia No $01678-2016$.

\section{ABSTRACT}

The judicialization so that the public health system provides medicines to patients in Costa Rica is increasingly frequent. The majority of these cases are resolved by the constitutional court. This article is a qualitative, analytical and descriptive study based on access to the drug Riociguat for the treatment of patients with thromboembolic pulmonary hypertension. All the resolutions on Riociguat for the Costa Rican case and an in-depth reference to judgment No. 01678 - 2016 are analyzed.

PALABRAS CLAVE: Judicialización, derecho, salud, medicamentos, derecho fundamental.

KEY WORDS: Judicialization, law, health, medicines, fundamental rights.

\footnotetext{
1 El presente artículo ha sido realizada en el marco del Proyecto de Investigación "La garantía constitucional al acceso de medicamentos en el sistema de salud costarricense".

2 Egresada en Sociología por la Universidad de Costa Rica, cristina.carpio@ucr.ac.cr. ORCID iD: https://orcid.org/0000-0003-3963-9904

3 Licenciado en Farmacia, Licenciado en Derecho, Especialista en propiedad intelectual de medicamentos, investigador del Instituto de Investigaciones Jurídicas de la Universidad de Costa Rica, freddy.arias_m@ucr.ac.cr. ORCID iD: https://orcid.org/0000-0003-2083-0004.
} 


\section{Introducción}

El Poder Judicial en Costa Rica está a cargo de la Corte Plena de Justicia dividida en el ámbito administrativo, auxiliar de justicia y jurisdiccional. Este último incluye los tribunales, juzgados y las Salas I, II, III y Constitucional ${ }^{4}$. La Sala Constitucional de la Corte Suprema de Justicia (en adelante Sala Constitucional) se crea por medio del artículo 10 de la Constitución Política de Costa Rica y la Ley de Jurisdicción Constitucional en Octubre del 1989 para conservar el principio de Supremacía constitucional. La máxima jerarquía con la que cuenta esta Sala le permite conocer las declaraciones de inconstitucionalidad de cualquier clase de normas jurídicas, de los actos sujetos al derecho público y dirimir procesos de competencia entre los poderes del estado ${ }^{5}$.

Desde la Convención Americana sobre Derechos Humanos, la salud es un bien jurídico supremo que busca ser conservada, promovida, protegida, recuperada y restaurada desde el Estado. La necesidad de protección efectiva frente a cualquier actuación que ponga en riesgo la salud psicológica, física o ambiental de las personas también coloca responsabilidad en las instituciones de salud como la Caja Costarricense del Seguro Social.

Por ello, los principios bajo los cuales actúan los órganos y entes públicos del servicio de salud han sido señalados desde la Sala Constitucional como adaptables a la eficiencia, eficacia, continuidad y regularidad, obligando a estos servicios a adaptarse de manera impostergable a las necesidades específicas de los pacientes, sobretodo en quienes demanden atención médica urgente, sin que se vean eximidos de su cumplimiento por carencia de recursos humanos o materiales. ${ }^{6}$

La protección de la salud de la población, al ser un deber del Estado, es susceptible de exigibilidad y para este fin existen instrumentos que lo materializan. El recurso de amparo es una garantía constitucional que permite la defensa de derechos fundamentales. Está amparado en el artículo 29 de la Ley de Jurisdicción Constitucional ${ }^{7}$. Este nace de la información que tiene el amparado, generalmente del médico, sobre la necesidad de un medicamento, y por un Comité Asesor Técnico Científico que lo negó, lo que origina que el paciente vea vulnerado su acceso a la salud (Primer Informe Estado de la Justicia, 2015: 21).

En este trabajo se analiza concretamente el caso del medicamento, Riociguat. Este medicamento generó treinta y nueve sentencias de la Sala Constitucional del año 2015 al año 2019 y en la última sección se brinda un análisis detallado de la sentencia № 01678 - 2016 con el fin de ejemplificar los supuestos que se trabajaron.

El Riociguat es un medicamento estimulador de la guanilato ciclasa soluble. Ayuda a regular el tono vascular, así como la proliferación e inflamación del músculo liso en la vasculatura pulmonar afectada por la hipertensión pulmonar. Es el único tratamiento que ha demostrado eficacia clínica significativa y sostenida en más de una forma de hipertensión pulmonar (HP).

En Costa Rica, es el único medicamento aprobado por el Ministerio de Salud para el trata-

4 Artículo 49 de la Ley Orgánica del Poder Judicial, la Corte Suprema de Justicia

5 Artículo 10 de la Constitución Política, de la Asamblea Nacional Constituyente

6 Sala Constitucional de la Corte Suprema de Justicia, STC 07193 - 2009, San José, a las catorce horas y veinte minutos del cinco de mayo del dos mil nueve

7 Ley 7135 de Jurisdicción Constitucional, de 11 de Octubre de 1989, La Gaceta, 34, 198 del 19 de Octubre de 1989. 
miento de pacientes con hipertensión pulmonar tromboembólica crónica. Basado en el estudio de Fase 3 CHEST (Ghofrani HA et al., 2013: 319-328), Riociguat es el primer tratamiento farmacológico aprobado para el tratamiento de pacientes con hipertensión pulmonar tromboembólica crónica (HPTEC) que no son candidatos a cirugía para pacientes con HP recurrente/persistente después del tratamiento quirúrgico. Riociguat también ha sido aprobado para el tratamiento de pacientes con hipertensión arterial pulmonar (HAP), para mejorar la capacidad de ejercicio, mejorar la clase funcional de la OMS y para retrasar el empeoramiento clínico. El estudio PATENT de Fase 3 y controlado por placebo mostró eficacia significativa de Riociguat en múltiples criterios de valoración en pacientes con HAP, tanto como monoterapia como en combinación con antagonistas de los receptores de la endotelina o análogos de prostaciclina. Hasta Riociguat, ninguna farmacoterapia específica había sido aprobada para el tratamiento de las formas no HAP de la HP.

La hipertensión pulmonar (HP) es un incremento patológico de la presión arterial pulmonar (PAP) asociado con varios padecimientos clínicos (Stasch and all, 2013). Aunque diferentes tipos de HP muestran distintas características fisiopatológicas, comparten varios procesos subyacentes: vasoconstricción, remodelación vascular (proliferación), trombosis y fibrosis en la vasculatura pulmonar, y todos están asociados con la disminución de los niveles del óxido nítrico (NO) endógeno (Ghofrani HA et al., 2013: 320).

Está investigación consiste en un análisis detallado de las sentencias emitidas como respuesta a casos de pacientes que alegan una violación a sus derechos fundamentales y solicitan que la CCSS les brinde el medicamento Riociguat.

\section{Metodología}

La estructura metodológica posee un enfoque cualitativo, analítico y descriptivo basado en el acceso al medicamento Riociguat. Este análisis de caso busca describir los datos que se encuentran en el texto y su exploración a profundidad desde varias perspectivas: el criterio del médico tratante, el criterio del Comité Central de Farmacología, el criterio de la Sección Clínica Médico Forense y el razonamiento jurídico de la Sala Constitucional.

La matriz completa utiliza una base de datos estática y de texto completo con un registro sistemático de datos, recolectados a partir de la literalidad de treinta y nueve sentencias disponibles en el sistema en línea del Poder Judicial "Nexus", que son el cuerpo completo de resoluciones de la Sala Constitucional emitidas entre 2015 y 2019 que circunscriben los procesos de Recursos Amparo en contra de la Caja Costarricense de Seguro Social (CCSS) en cuanto al acceso de Riociguat. Instrumentalmente se crea una base de datos que facilita el análisis completo, en conjunto y al mismo tiempo disminuye la posibilidad de pérdida o reiteración de datos.

La base de datos contiene las siguientes categorías generales: 1) año de emisión, 2) número de sentencia, 3) número de expediente 4) la resolución del caso sea con lugar, sin lugar, parcialmente con lugar, u otras. También se extrajeron descriptores de contenido de sentencia como: 5) existencia de conflicto por despacho de medicamentos, y por último; se individualizó y categorizó los criterios del médico tratante, del Comité Central de Farmacología o Comité Local de Farmacoterapia según el caso y de la Sección Clínica Médico Forense. 


\section{Resultados}

En la figura 1 se muestra la distribución de las 39 sentencias que se referían al Riociguat. La primer resolución que se emitió en el país fue en año 2015, luego aumentó a siete en 2016, cinco en el 2017 y trece en el año 2018 y 2019. Durante los cinco años, las sentencias emitidas han sido declaradas con lugar a favor del recurrente, exceptuando una sentencia declarada sin lugar en el año 2016.

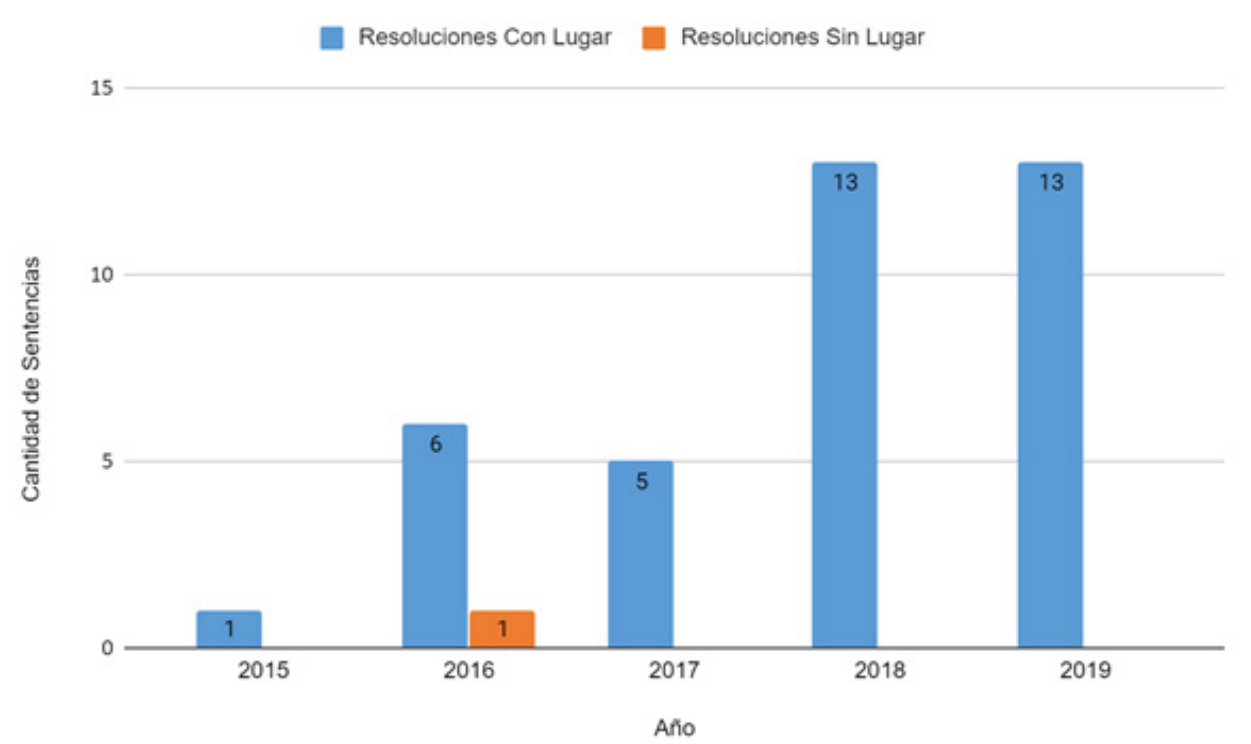

Figura 1. Resoluciones de sentencias de la Sala Constitucional de casos por Riociguat, del año 2015 a 2019 Fuente: Elaboración propia.

\section{Discusión}

Del análisis de las sentencias emitidas desde 2015, es posible determinar la dinámica de los sujetos procesales, incluyendo las partes, terceros e intervinientes, pero para efectos de este análisis se profundiza en las actuaciones y criterios de: i) el médico tratante, ii) el Comité Central de Farmacoterapia, iii) la Sección Clínica Médico Forense y iv) la Sala Constitucional.

El escenario de análisis contiene una dinámica interna del conflicto, que se genera por el contraste entre las pretensiones de las personas amparadas, que se basa en la prescripción del médico tratante, y que están en contradicción con las resoluciones del Comité Central de Farmacoterapia; al que se le suma el criterio de la Sección Clínica Médico Forense.

A continuación, se profundiza cada uno de los criterios que se contraponen en el proceso de resolución sobre medicamentos que realiza la Sala Constitucional. 


\section{Del médico tratante}

El proceso administrativo en la CCSS para brindar un medicamento que está fuera de la LOM requiere que el médico que trata directamente al paciente, se responsabilice por la prescripción. Desde los primeros casos relacionados con el despacho del Riociguat, en 2015, el criterio del médico tratante se ha basado en los beneficios y mejoras del medicamento sobre la calidad de vida de los pacientes. Los estudios con alto nivel de evidencia científica han sido el fundamento principal para la prescripción del Riociguat en los pacientes con hipertensión pulmonar. Esta enfermedad afecta el músculo esquelético y se caracteriza por una presión elevada (hipertensión, mayor a $25 \mathrm{mmHg}$ ) en los vasos que llevan la sangre desde el corazón a los pulmones (arterias pulmonares) para que se oxigene produciendo un déficit de sustancias que dilatan los vasos y aumentan las sustancias que los contraen, por lo que el corazón debe trabajar más fuerte para oxigenar al organismo. La sintomatología abarca cansancio, disnea, falta de aire, desmayos y dolor en el pecho.

El criterio del médico tratante plantea al Riociguat como el mejor tratamiento para la hipertensión pulmonar. Esta posición ha sido reiterada en los casos del 2015, 2016, 2017, 2018 y 2019 de forma constante al argumentar que existe literatura que sustenta su utilización ${ }^{8}$. Se puede determinar que el criterio de los médicos tratantes a través de los años se homologa en una inclinación por el uso del Riociguat, pues contemplan la evidencia científica de estudios clínicos de fase 3 , concretamente CHEST y PATENT ${ }^{9}$, demostrado una mejoría en síntomas y calidad de vida de las personas.

\section{Del Comité Central de Farmacología}

El Comité Central de Farmacoterapia es un órgano colegiado que se encarga de seleccionar y velar por la seguridad, calidad y uso eficiente de los medicamentos en la Caja. Es un órgano técnico creado por la Caja Costarricense de Seguro Social, de conformidad con el Decreto Ejecutivo № 19343-S del 23 de noviembre de 1989. Tiene una integración multidisciplinaria de profesionales de ciencias de la salud, la función esencial consiste en confeccionar el listado básico o lista oficial de medicamentos de la Caja con normas administrativas y de información concordantes con el Formulario Terapéutico Nacional; velar por la correcta aplicación de la Lista Oficial de Medicamentos emitida por la Caja, y la aprobación de la compra de productos farmacéuticos que no estén incluidos en ella ${ }^{10}$. Es un órgano colegiado, de carácter permanente, asesor y adscrito a la Gerencia Médica de la Caja Costarricense de Seguro Social, responsable de la toma de decisiones en aspectos de política de medicamentos y farmacoterapéuticos en la institución. ${ }^{11}$

El criterio que adopta este órgano no está determinado por un individuo, sino por un grupo de profesionales en la materia que desarrollan sus posicionamientos con la finalidad de brindar criterio técnico y médico a sus actuaciones y decisiones.

\footnotetext{
8 En la resolución $N^{\circ}$ 02252-2016: "La médico tratante de la amparada, Dra. Rocha Contreras del Servicio de Neumología del Hospital México, le prescribió el medicamento Riociguat, para tratar su enfermedad, porque representa una de las mayores innovaciones farmacológicas en los años recientes. La estimulación directa de la GCs aunada a la sensibilización del NO endógeno, es un nuevo mecanismo de acción que se enfoca en regular al eje NO-GCsGMPc comprometido y a la disfunción endotelial en la HP (Evgenov OV, Nat Rev Drug Discov 2006;5:755-68)."

9 Hossein-Ardeschir, G, D'Armini M. \& Gerrit W, 2013, Riociguat for the treatment of chronic thromboembolic pulmonary hypertension. The New England Journal of Medicine \& Rubin, Lewis J., Galiè, N. And Hossein \& Ardeschir G., 2015, Riociguat for the treatment of pulmonary arterial hypertension: a longterm extension study (PATENT-2). The European Respiratory Journal

10 Reglamento 8314 del Comité Central de Farmacoterapia de la Caja Costarricense de Seguro Social, de 15 de Enero de 2009, Gaceta, 29, del 11 de Febrero de 2009 11 Reglamento 8314 del Comité Central de Farmacoterapia de la Caja Costarricense de Seguro Social, de 15 de Enero de 2009, Gaceta, 29 , del 11 de Febrero de 2009.
} 
El Comité Central de Farmacoterapia ha considerado que no existe suficiente evidencia científica de los beneficios del Riociguat, pues considera que sus resultados son muy limitados para autorizar su uso en los pacientes remitidos. Esta posición se ha mantenido durante el 2015, 2016 y 2017 como la única línea discursiva en las sentencias, disminuyendo durante el 2018 a un $77 \%$ y 2019 a un $85 \%$.

Durante los últimos años, 2018 y 2019, el Comité Central de Farmacología complementa su criterio al argumentar que las peticiones de uso para Riociguat no son posibles de tramitar, pero asegura que esta decisión no vulnera el derecho de acceso a la salud en los pacientes, pues existen alternativas que se encuentran en la Lista Oficial de Medicamentos (LOM) que sí pueden brindarse; y a falta de estos o impedimentos para el paciente, apoyan el uso de otros medicamentos que no están en la LOM que han demostrado ser superiores al Riociguat y son apoyadas por la Caja Costarricense del Seguro Social (CCSS).

El posicionamiento del Comité Central de Farmacología y los criterios de los diferentes médicos tratantes resultan antagónicos ante la discusión principal que es el tratamiento ideal y efectivo de los pacientes con hipertensión pulmonar.

\section{De la Sección Clínica Médico Forense}

La Sección Clínica Médico Forense fue creada en el 26 de abril de 1974, bajo la ley 5529 y actualmente está contenida en la Ley Orgánica 5524 del Organismo de Investigación Judicial (OIJ), su objetivo es brindar información científica y objetiva por medio de valoraciones sobre el estado físico de las personas vivas, hallazgos médicos y su relación con la investigación para contribuir con la Autoridad Judicial que lo solicita. La Dirección del Organismo de Investigación Judicial, cuenta con tres departamentos: $1^{\circ}$ ) Departamento de Investigaciones Criminales; $2^{\circ}$ ) Departamento de Medicina Legal y $3^{\circ}$ ) Departamento de Laboratorios de Ciencias Forenses ${ }^{12}$. El Departamento de Medicina Legal es un grupo de especialistas en medicina legal o médicos especializados en otras ramas encargados de efectuar los exámenes y evacuar las respectivas consultas médico-forenses, en los casos cuyo conocimiento corresponda al Organismo. Dentro de este último se encuentra contenido la Sección Clínica Médico Forense que se conforma de una jefatura y doce médicos forenses que en promedio realizaron servicios a doce mil personas usuarias durante los años 2016 a 2018 (Vega et al, 2019, 141-142).

El análisis realizado de la Sección Clínica Médico Forense desde el 2016 al 2019 concluyó en un $92 \%$ de los casos totales que la terapia con Riociguat es la mejor opción y que existe evidencia científica para demostrar la mejoría en síntomas y calidad para el paciente. El restante $8 \%$ corresponde al caso del 2015 donde no participaba como sujeto procesal necesario y dos casos del 2016 en donde no se indica su participación, específicamente en las sentencias 02252-2016 y 07036-2016.

En la sentencia 02252-2016, se presentó un caso de una paciente que tiene intolerancia al medicamento lloprost y Sildenafil, por lo que su médico tratante solicitó la autorización para administrar Riociguat, solicitud que fue negada por el Comité Central de Farmacoterapia. La Sala Constitucional consideró que la petición de denegatoria del medicamento es contraria al criterio de la médico tratante y del Jefe del Servicio de Neumología, y por tanto es ilegítima y lesiva de los derechos fundamentales de la paciente; dando con lugar 
el recurso, sin solicitar o justificar la ausencia de la Sección Clínica Médico Forense ${ }^{13}$. En la sentencia 07036-2016 la Sala Constitucional ante la divergencia de criterios entre el médico tratante y el Comité Central de Farmacoterapia y en aras de proteger la salud y la calidad de vida falló a favor del paciente; agregando que al menos en cuatro ocasiones el tribunal constitucional ha avalado el uso del medicamento y por tanto no es necesario remitir al médico forense. ${ }^{14}$

\section{De la Sala Constitucional de la Corte Suprema de Justicia}

Durante el 2015 la Sala Constitucional señaló la libertad de prescripción médica con el objetivo de respaldar un mejor pronóstico y la calidad de vida para el paciente. El criterio del médico tratante ha prevalecido en reiteradas sentencias de la Sala Constitucional como en las resoluciones 02252-2016, 07036-2016 y 06440-2018, desarrollando ampliamente su conceptualización como la capacidad o posibilidad de brindar al paciente un mejor pronóstico y una mejor calidad de vida. Para este tribunal, el médico tiene un conocimiento más cercano con el paciente, interpretando dentro de este criterio la protección de la vida, la salud de las personas y la tutela obligatoria del Estado al brindar servicios públicos y suministrar medicamentos. El médico mantiene una libertad e independencia profesional la cual es necesaria para atender a los pacientes, elaborar sus diagnósticos y prescribir tratamientos con la mejor asistencia posible. ${ }^{15}$

Desde el año 2016 la Sala ha integrado la jerarquización de intereses económicos y el derecho a la salud, dejando claro que el derecho a la vida y la salud no pueden ser suspendidos o disminuidos a razón de criterios económicos. La vida y el acceso a condiciones mínimas de salud son la base del resto de derechos fundamentales, además los costos directos e indirectos de tal incapacidad incluyen afectaciones sociales y morales mucho mayores $^{16}$.

El derecho a la salud se ha desarrollado en las sentencias 17500-2017 y 05472-2016 como un derecho fundamental autónomo con relación al numeral 21 constitucional sobre el derecho a la vida, siendo este último la base para los demás derechos fundamentales, y con ello el retardo en los hospitales, clínicas y demás unidades de la Caja Costarricense del Seguro Social repercute negativamente en la protección de la salud y vida de los usuarios ${ }^{17}$.

En la sentencia 05472-2016 la Sala Constitucional señaló que el acceso a los servicios de salud conlleva: la accesibilidad a servicios, la no discriminación, la accesibilidad física, económica y equitativa, por esto los criterios económicos que brinda el Comité Central de Farmacoterapia pueden llevar razón al negar el medicamento, pero al contraponerse con el acceso a la salud pierde su respaldo.

Desde el 2017, en las sentencias 03573-2017, 21717-2018 y 23656-2019, la Sala ha disminuido la amplitud de los criterios bajo los cuales actuó, limitándose a mencionar en un

\footnotetext{
13 Sala Constitucional de la Corte Suprema de Justicia, STC 02252-2016, , San José de las quince horas quince minutos del dieciséis de febrero de dos mil dieciséis

14 Sala Constitucional de la Corte Suprema de Justicia, STC 07036-2016, San José del a las catorce horas treinta minutos del veinticuatro de mayo de dos mil dieciséis

15 Sala Constitucional de la Corte Suprema de Justicia, STC 05355-2015, San José a las nueve horas cinco minutos del diecisiete de abril de dos mil quince 16 Sala Constitucional de la Corte Suprema de Justicia, STC 05472-2016, San José a las quince horas cinco minutos del veintiséis de abril de dos mil dieciséis 17 Sala Constitucional de la Corte Suprema de Justicia, STC 17564-2017, San José, a las nueve horas quince minutos del tres de noviembre de dos mil diecisiete.
} 
párrafo de la resolución la solicitud de medicamentos, así como su relación con el criterio del médico tratante y su prevalencia ante criterios económicos. Posteriormente, en las sentencias 16055-2018 y 22824-2019 se mencionó la jurisprudencia del médico tratante y su prevalencia como forma de mantener la vida y la salud de las personas.

\section{Resolución Nº 01678 - 2016}

Esta resolución ${ }^{18}$ ilustra un caso que fue analizado por la Sala Constitucional con una sentencia que rechazó el medicamento prescrito por el médico tratante. Se planteó una discusión respecto a la evidencia científica en el uso del medicamento Riociguat. Es el único caso en que se ha rechazado un recurso de amparo presentado respecto al uso de este medicamento.

El caso trata de un adulto de 37 años de edad y que padecía de hipertensión pulmonar tromboembólica, clase funcional IV o hipertensión pulmonar tromboembólica crónica, desde hace ocho años y fue tratado en el Hospital México. Manifestó que esta enfermedad es severa y no tiene cura, pero con tratamiento adecuado podría tener una mejor calidad de vida, ya que, le controlaría los síntomas y le previene un daño mayor a sus pulmones. Actualmente, toma el medicamento Sildenafil. La médica tratante, señaló que para este padecimiento la cirugía sería lo mejor, pero es de alto riesgo, por lo que le recetó el medicamento denominado Riociguat.

Ante este caso, la Sala Constitucional realizó el siguiente análisis:

"II.- Caso concreto.- La recurrente presenta recurso de amparo y manifiesta que el amparado padece de varias enfermedades, entre ellas, hipertensión pulmonar tromboembólica, clase funcional IV desde hace 8 años y es tratado en el hospital recurrido. Manifiesta que esta enfermedad es severa y no tiene cura, pero con tratamiento adecuado podría tener una mejor calidad de vida, ya que, le controlaría los síntomas y le previene un daño mayor a sus pulmones. Acota que la Doctora Betty Rocha Contreras, médico tratante, señaló que para este padecimiento la cirugía sería lo mejor, pero es de alto riesgo, por lo que le recetó el medicamento denominado Riociguat. Sin embargo, el Comité Central de Farmacoterapia acordó en la sesión No. 2015-51, no avalar el tratamiento solicitado. Por su parte, las autoridades de la Caja Costarricense de Seguro Social informan bajo juramento que la decisión de rechazar el medicamento para el amparado, no es antojadiza, pues todo lo contrario, el accionar de la Caja Costarricense de Seguro Social y el proceso de Selección de Medicamentos para atender necesidades excepcionales, en todos los casos, busca prevalecer el beneficio al paciente objetivamente evaluado según el paradigma de la medicina basada en evidencia, evitando el subjetivismo, los conflictos de interés y la confusión derivada de la promoción comercial. Y en el caso específico del amparado, como se ha expuesto, dentro del protocolo vigente en la institución, se apoya el uso de inhibidores de la fosfodiesterasa como el Sildenafil; sin embargo, la médica prescriptora solicita el Riociguat debido a que el paciente se encuentra en un estadio funcional III, pero, aduce que sólo el Riociguat es utilizable en el caso del amparado, lo cual es falso, ya que el Sildenafil es una alternativa terapéutica que ofrece una evidencia científica más robusta y que ha sido comparado de forma directa contra otros fármacos en múltiples estudios. Ante esa disyuntiva, este Tribunal solicitó el criterio técnico de la Sección Clínica Médico Forense y en lo conducente ésta autoridad en el informe rendido a la Sala indicó: "...Se recomienda que los casos que persisten sintomáticos con la terapia inicial que corresponde a lo descrito previamen-

18 Sala Constitucional de la Corte Suprema de Justicia, STC 001678-2016, San José, a las nueve horas cuarenta minutos del tres de febrero de dos mil dieciséis 
te, se pueden hacer las siguientes combinaciones: riociguat al bosentan, selexipag a un antagonista del receptor de endotelina y/o inhibidores de la fosfodiesterasa o agregar sildenafil a epoprostenol. Lo anterior con recomendación y nivel de evidencia I de la Sociedad Europea de Cardiología / Sociedad Respiratoria Europea (...) de acuerdo a la revisión de la medicina basada en la evidencia, para el estado actual del evaluado catalogado por sus médicos tratantes con hipertensión pulmonar tromboembólica crónica inoperable, lo recomendado en la actualidad es hacer una combinación de medicamentos cuando ha fallado la terapia inicial, como se ha especificado en la fundamentación Médico Legal...". macitentan al sildenafil, riociguat al bosentan, selexipag a un antagonista del receptor de endotelina y/o inhibidores de la fosfodiesterasa o agregar sildenafil a epoprostenol. Lo anterior con recomendación y nivel de evidencia I de la Sociedad Europea de Cardiología I Sociedad Respiratoria Europea (...) de acuerdo a la revisión de la medicina basada en la evidencia, para el estado actual del evaluado catalogado por sus médicos tratantes con hipertensión pulmonar tromboembólica crónica inoperable, lo recomendado en la actualidad es hacer una combinación de medicamentos cuando ha fallado la terapia inicial, como se ha especificado en la fundamentación Médico Legal...". (Sala Constitucional de la Corte Suprema de Justicia, STC 001678-2016)

Bajo esta perspectiva, el Tribunal en el asunto bajo estudio, no acreditó prueba suficiente para acoger el reclamo de la recurrente, y desvirtuar la tesis adoptada por las autoridades recurridas, pues las autoridades forenses no recomiendan para beneficio del amparo el medicamento indicado por la médico prescriptora. En consecuencia, la Sala no verificó que la negativa de las autoridades recurridas del Comité Central de Farmacoterapia de suministrar el citado medicamento Riociguat al amparado, esté lesionando el derecho a la salud del paciente. Por tanto, se declaró sin lugar el recurso.

Algunos aspectos importantes en la resolución del caso es que los elementos que considera el Tribunal Constitucional se limitan a transcribir los planteamientos de las diferentes instancias, sin analizar detalladamente cada uno de los argumentos. La Sala Constitucional respalda un criterio basado, supuestamente, en evidencia científica, sin embargo, esta evidencia no existe y es fácilmente constatable. Basta con revisar las indicaciones aprobadas de los medicamentos por la autoridad competente para establecer la evidencia científica que respalda el uso de un medicamento.

Del análisis de la normativa nacional es claro que las potestades del Comité Central de Farmacoterapia están limitadas por las disposiciones del Ministerio de Salud, en ejercicio de sus competencias de autoridad sanitaria. Ninguna disposición del Comité puede sobrepasar la autoridad del Ministerio de Salud respecto al uso de medicamentos. Siempre que el CCF pretenda incluir en sus guías de farmacoterapia cualquier medicamento o indicación está limitado a la autorización del Ministerio de Salud.

El registro sanitario de un medicamento es un procedimiento administrativo obligatorio, es la clave para ejercer un control legal y preventivo de los medicamentos con el fin de velar por su calidad, seguridad, eficacia y uso correcto. Con ese propósito se evalúa la información necesaria que el solicitante del registro, legalmente capacitado para pedirlo, debe proporcionar a la autoridad y que se refiere esencialmente a información científica y técnica sobre el producto y datos administrativos generales.

La información aportada por el solicitante es revisada detalladamente por los profesio- 
nales que se aseguran de la seguridad, eficacia y calidad de los productos. Una vez que se otorga esta autorización, los productos se pueden comercializar en el país.

El fundamento de este procedimiento es que se materialice la obligación que tiene el Estado, por medio del Ministerio de Salud de asegurar que los productos son adecuados para el consumo humano. Además, que el fabricante del producto haya cumplido con las regulaciones nacionales e internacionales para que el producto no signifique un riesgo para la salud de la población.

En el caso de medicamentos, el registro se realiza siguiendo lo dispuesto en una normativa común centroamericana, el Reglamento Técnico Centroamericano 11.03.59:11 ${ }^{19}$. Este Reglamento establece los requisitos mínimos que debe presentar el fabricante para que se otorgue la autorización de uso.

La información contenida en los registros sanitarios es de gran importancia, porque contempla la información de las indicaciones, contraindicaciones, interacciones, efectos adversos, dosis y administración. La obtención de este permiso limita la utilización que se le puede dar a un medicamento, pues no se puede prescribir el medicamento para indicaciones que no hayan sido aprobadas por la autoridad sanitaria, al igual que la compañía que registra el medicamento no puede promover su uso en indicaciones para las que no tenga permiso.

Es posible que un medicamento sea autorizado para un determinado uso en el país, pero esto no quiere decir que se autoriza su uso para cualquier enfermedad, sino que cada indicación de uso debe ser autorizada por el Ministerio de Salud. Este trámite administrativo es realizado también por la Unidad de Registros. El fundamento de este proceso es el artículo 115 de la Ley General de Salud.

En el caso que motivó la sentencia número 01678-2016, se genera una discusión sobre un medicamento que la CCSS le brinda sin que tenga indicaciones para utilizarse para el tratamiento de esta enfermedad en el país, a pesar de esto es defendido en múltiples ocasiones.

En el análisis del caso analizado por la Sala Constitucional es claro que el uso del medicamento Sildenafil es absolutamente incorrecto al no estar autorizado en el país, y no se respalda en evidencia ciekntífica para tratar un paciente con hipertensión pulmonar tromboembólica crónica.

El criterio brindado por la Sección Clínica médico forense indica esquemas de tratamiento que no han sido aprobados en el país y no reconoce la evidencia científica que existe para el único medicamento aprobado por el Ministerio de Salud para hipertensión pulmonar tromboembólica crónica.

En el análisis del caso nunca se considera la evidencia científica que fundamenta la obtención de un registro sanitario y a su vez se utilizan interpretaciones incorrectas relativas a la evidencia que existe respecto del medicamento. 


\section{Conclusiones}

La resolución de la Sala Constitucional de recursos de amparo por acceso al medicamento Riociguat aumenta cada año. El Tribunal Constitucional condena a la Caja Costarricense de Seguro Social ante la renuencia de otorgar este medicamento a los pacientes. Desde el año 2015 a 2019 se declararon con lugar 38 de los 39 casos presentados por los pacientes. Este resultado consistente debe servir para que la CCSS analice sus procedimientos internos y tome las medidas administrativas necesarias para facilitar el acceso de los medicamentos a los ciudadanos y evite la judicialización de estos casos. Por su parte la Sala Constitucional debe analizar el contenido del término "medicina basada en la evidencia" y analizar detalladamente su significado y fundamento para utilizarla como base de sus resoluciones.

\section{REFERENCIAS BIBLIOGRÁFICAS}

- Hossein-Ardeschir, G, D'Armini M. \& Gerrit W. (2013). Riociguat for the treatment of chronic thromboembolic pulmonary hypertension. The New England Journal of Medicine, 369, 319-329. Disponible en: https://www.nejm.org/doi/full/10.1056/nejmoa1209657.

- Programa Estado de la Nación. (2015). Judicialización de la salud: revisión de los recursos de amparo relacionados con medicamentos. (Primer Informe Estado de la Justicia) San José: Programa Estado de la Nación.

- Rubin, Lewis J., Galiè, N. And Hossein \& Ardeschir G. (2015). Riociguat for the treatment of pulmonary arterial hypertension: a long-term extension study (PATENT-2). The European Respiratory Journal, 45, 1303-1313. Disponible en: https://erj.ersjournals. com/content/erj/45/5/1303.full.pdf.

- Stasch, J. P \& Oleg V., E. (2013). Soluble guanylate cyclase stimulators in pulmonary hypertension. Handbook of experimental pharmacology, 218, 279-313. Disponible en: pubmed.ncbi.nlm.nih.gov/24092345/ .

- Vega Zúñiga, F., Chacón Barquero, L. y Villalobos León, K. (2019). Medicina Legal de Costa Rica Historia, actualidad y proyección a futuro. Revista Medicina Legal en Costa Rica 36, 2, 127-165. Disponible en: https://www.scielo.sa.cr/pdf/mlcr/v36n2/22155287-mlcr-36-02-127.pdf.

Normativa y Jurisprudencia citada

- Asamblea Nacional Constituyente. Constitución Política, de 7 de noviembre de 1949.

- Asamblea Legislativa de Costa Rica. Ley Orgánica 5524, de 7 de Mayo de 1974, del

Organismo de Investigación Judicial, (Colección de Leyes y Decretos 1 (2): 979, 9 de Julio de 1973).

- Asamblea Legislativa de Costa Rica. Ley 7135, de 11 de Octubre de 1989, de Jurisdicción Constitucional (La Gaceta 34, 198 del 19 de Octubre de 1989).

- Asamblea Legislativa de Costa Rica. Reglamento 8314, de 15 de Enero de 2009, del

Comité Central de Farmacoterapia de la Caja Costarricense de Seguro Social (Gaceta 29, del 11 de Febrero de 2009).

- Poder Ejecutivo. Decreto Ejecutivo 38414, del 28 de Febrero de 2014, COMEX-MEIC-S (Gaceta 103, 20, del 30 de Mayo de 2014). 
Jurisprudencia citada

- Sala Constitucional de la Corte Suprema de Justicia, STC 07193 - 2009, San José, a las catorce horas y veinte minutos del cinco de mayo del dos mil nueve.

- Sala Constitucional de la Corte Suprema de Justicia, STC 05355-2015, San José a las nueve horas cinco minutos del diecisiete de abril de dos mil quince.

- Sala Constitucional de la Corte Suprema de Justicia, STC 001678-2016 San José, a las nueve horas cuarenta minutos del tres de febrero de dos mil dieciséis.

- Sala Constitucional de la Corte Suprema de Justicia, STC 02252-2016, San José de las quince horas quince minutos del dieciséis de febrero de dos mil dieciséis.

- Sala Constitucional de la Corte Suprema de Justicia, STC 05472-2016, San José de las quince horas cinco minutos del veintiséis de abril de dos mil dieciséis.

- Sala Constitucional de la Corte Suprema de Justicia, STC 07036-2016, San José del a las catorce horas treinta minutos del veinticuatro de mayo de dos mil dieciséis.

- Sala Constitucional de la Corte Suprema de Justicia, STC 17564-2017, San José, a las 